\title{
Revista Brasileira de Enfermagem REBEn \\ Avaliação da satisfação de pacientes oncológicos com atendimento recebido durante o tratamento antineoplásico ambulatorial
}

\author{
Evaluation of the satisfaction level of cancer patients with the assistance recieved during \\ ambulatory antineoplastic chemoteraphy \\ Evaluación de la satisfaccion de pacientes oncológicos con la atención recibida durante \\ el tratamiento antineoplásico ambulatorial
}

\section{Selma Montosa da Fonseca}

Enfermeira. Doutora em Ciências. Gerente de Enfermagem das Clínicas Médicas Especializadas do Hospital São Paulo/ UNIFESP, São Paulo, SP.

\section{Maria Gaby Rivero de Gutiérrez}

Enfermeira. Doutora em Enfermagem. Professora Adjunto do Departamento de Enfermagem da UNIFESP, São Paulo, SP.

Orientadora.

\section{Nilce Piva Adami}

Enfermeira. Doutora em Saúde Pública. Professora Titular do Departamento de Enfermagem da UNIFESP, São Paulo, SP.

Co-orientadora.

Parte da tese apresentada a UNIFESP em 2006.

\section{RESUMO}

Este estudo descritivo, fundamentado na abordagem de resultados proposta por Donabedian, teve como objetivo avaliar o nível de satisfação de pacientes oncológicos com o atendimento recebido no Ambulatório de Quimioterapia de Adultos do Hospital São Paulo. A amostra incluiu 105 pacientes que aceitaram participar do estudo. A avaliação dos usuários foi positiva, tanto com o atendimento de enfermagem $(54 \%$ muito bom e $46 \%$ bom), quanto com o atendimento global do serviço (50\% muito satisfeitos e $46 \%$ satisfeitos). 0 nível de satisfação manifestado pelos usuários relacionou-se, principalmente, com a acessibilidade organizacional, o ambiente acolhedor e o processo assistencial nas dimensões da interação profissional/cliente e do desempenho técnico. No entanto, apontaram como medidas necessárias, evitar a falta de drogas e aumentar o número de vagas.

Descritores: Satisfação do paciente/Quimioterapia; Avaliação em enfermagem; Enfermagem Oncológica.

\section{ABSTRACT}

This descriptive study, grounded upon Donabedian's outcomes approach, had as purpose to evaluate the satisfaction level of cancer patients with the assistance received at the Adult Chemotherapy Ambulatory of Hospital São Paulo. The sample was constituted of 105 patients who accepted to participate in the study. The patients' evaluation was positive both with nursing care $(54 \%$ very good and $46 \%$ good) and with the overall care received in that service (50\% very satisfied and $46 \%$ satisfied). The level of satisfaction manifested by the clients was related mainly to the organization accessibility, the welcoming environment and the assistance process, in its professional/client interaction and the technical performance dimensions. However, they also pointed out the need for measures aimed at preventing drugs shortage and the lack of vacancies for new patients.

Descriptors: Patient satisfaction/Chemotherapy; Nursing assessment; Oncologic nursing.

\section{RESUMEN}

Este estudio descriptivo, fundado en el abordaje de resultados propuesto por Donabedian, tuvo como objetivo evaluar el nivel de satisfacción de pacientes con cáncer con la atención recibida en el Ambulatorio de Quimioterapia de Adultos de Hospital São Paulo. La muestra incluyó 105 pacientes que aceptaron participar de la investigación. La evaluación de los usuarios fue positiva, tanto con la atención de enfermería (54\% muy buena y $46 \%$ buena), cuanto con la atención general del servicio (50\% muy satisfechos y $46 \%$ satisfechos). El nivel de satisfacción manifestado por los usuarios se relacionó, principalmente, con la accesibilidad organizacional, el ambiente acogedor y el proceso asistencial en las dimensiones de la interacción profesional/cliente y del desempeño técnico. No obstante, apuntaron como medidas necesarias, evitar la falta de medicinas y aumentar el número de plazas.

Descriptores: Satisfacción del paciente/Quimioterapia; Evaluación de enfermería; Enfermería Oncológica.

Fonseca SM, Gutiérrez MGR, Adami NP. Avaliação da satisfação de pacientes oncológicos com atendimento recebido durante o tratamento antineoplásico ambulatorial. Rev Bras Enferm 2006 set-out; 59(5): 656-60.

\section{INTRODUÇÃO}

As organizações hospitalares têm buscado avaliar a qualidade dos serviços prestados a fim de aperfeiçoar os seus processos de trabalho e, para satisfazer as necessidades dos seus clientes, devem disponibilizar canais para ouvi-los, considerando suas expectativas como uma bússola que direciona as mudanças ${ }^{(1)}$.

A busca da qualidade caracteriza-se pelo desenvolvimento de um processo contínuo de melhoria das práticas desenvolvidas em um serviço, tendo em vista o aprimoramento do atendimento prestado aos seus usuários ${ }^{(2,3)}$. 
Segundo as funções a cumprir, a avaliação formativa visa fornecer informações para adequar e superar aspectos problemáticos de um programa em andamento e volta-se para o desenvolvimento de intervenções, mediante a perspectiva do cliente. As abordagens participativas, centradas nos usuários, têm como objetivo engajá-los no processo de avaliação, para que os resultados sejam considerados nas estratégias de aprimoramento dos serviços de acordo com os pressupostos dos avaliadores de $4^{\mathrm{a}}$ geração( ${ }^{(3)}$.

Apesar das experiências consolidadas, a partir do início do século XX, nos Estados Unidos e Canadá, o despertar pelo tema qualidade da assistência à saúde em âmbito internacional teve visibilidade nos últimos anos desse século, impulsionado por diversas razões, tais como: aumento das demandas por cuidado de saúde; custos crescentes para manutenção dos serviços de saúde e limitados recursos disponíveis; evidências da variação na prática clínica; usuários mais exigentes. Estes fatos incrementaram a implementação dos programas de garantia de qualidade em instituições hospitalares, principalmente do setor privado, ancorados no referencial utilizado para indústrias, no qual o cliente é considerado a figura principal da definição de qualidade. No Brasil, as primeiras iniciativas independentes de acreditação hospitalar ocorreram no início de 1990 e, em 1996, o Programa Brasileiro de Qualidade e Produtividade priorizou o Projeto de Avaliação e Certificação dos Serviços de Saúde, sendo que em 1998 foi lançado pelo Ministério da Saúde o Programa Brasileiro de Acreditação Hospitalar(4).

A avaliação da qualidade da assistência prestada é imprescindivel para o planejamento e gerenciamento dos sistemas de saúde. A mensuração da satisfação do paciente, a partir da utilização dos serviços de saúde, é um dos componentes dos resultados desejáveis da assistência prestada. No entanto, apresenta limitações como indicador de qualidade pela sua subjetividade, fazendo parte do grupo de indicadores de imagem das instituições de saúde que trabalham principalmente com resultados de pesquisa acerca da satisfação dos clientes externos e internos ${ }^{(5,6)}$.

Tendo em vista que os estudos avaliativos realizados sobre o processo assistencial de enfermagem desenvolvido no Ambulatório de Quimioterapia de Adultos do Hospital São Paulo (HSP) da Universidade Federal de São Paulo (UNIFESP) foram realizados sob a ótica dos profissionais envolvidos nesse processo, considerou-se essencial conhecer a percepção dos seus usuários, a fim de que mudanças a serem implementadas contemplassem suas expectativas.

Também, o expressivo aumento da demanda por atendimento nesse Ambulatório e as situações conjunturais devidas à crise financeira de hospitais universitários, reforçaram a necessidade de se reavaliar a qualidade do atendimento prestado.

Desse modo, o objetivo geral desta pesquisa foi: avaliar a satisfação de pacientes com câncer submetido à quimioterapia, com o atendimento recebido, por meio da verificação da sua satisfação com: a acessibilidade organizacional ao cuidado e com o ambiente do tratamento; o processo assistencial nas dimensões da interação profissional/cliente e do aspecto técnico do cuidado; os resultados do atendimento, expressos pela sua aderência ao tratamento, número de hospitalizações por complicações relacionadas aos efeitos adversos da quimioterapia e verbalização sobre os benefícios auferidos com as informações e cuidados recebidos.

\section{MÉTODO}

Trata-se de estudo avaliativo, descritivo, que analisou, segundo a abordagem de resultado, a satisfação dos clientes no Ambulatório de Quimioterapia de Adultos do HSP, no período de junho a outubro de 2004. Este hospital foi credenciado como Centro de Alta Complexidade em Oncologia (CACON I) e sua área de cobertura atende o Núcleo 5 da Secretaria de Estado da Saúde de São Paulo que abrange uma área extensa, incluindo bairros das zonas Sul e Leste da cidade de São Paulo

Atendendo à Resolução n 196/96 do Conselho Nacional de Saúde, este projeto foi aprovado pelo Comitê de Ética em Pesquisa da UNIFESP, sob n ${ }^{\circ}$

\section{0/03.}

Considerando que dentre as estratégias de coleta de dados, nem sempre as intervenções individuais são as mais adequadas para que o cliente possa expressar livremente suas opiniões, selecionou-se a intervenção grupal denominada técnica do grupo focal( ${ }^{(7)}$.

Porém, a adoção, nesse Ambulatório, de vários esquemas de tratamento, que apresentam periodicidades diferentes, dificultou o agendamento dos pacientes para a realização de oito encontros, impossibilitando assim, a aplicação dessa técnica. Optou-se, então, pela coleta de dados, por meio de entrevista semi-estruturada com os usuários do serviço.

Para tanto, construiu-se um instrumento fundamentado, principalmente no trabalho de Sitzia e Wood, e validado por sete especialistas, para avaliar a satisfação do paciente a respeito de componentes da estrutura, processo e resultados, conforme proposto por Donabedian ${ }^{(6,8)}$.

Aamostra foi constituída por 105 usuários do Ambulatório, representando $30 \%$ do número de atendimentos mensais, com qualquer tipo de câncer, que estavam, pelo menos, no sexto ciclo de quimioterapia e em condições clínicas para serem entrevistados por uma enfermeira não participante da equipe desse serviço e, que concordaram em participar da pesquisa mediante assinatura do Termo de Consentimento Livre e Esclarecido. Ainclusão de pacientes a partir desse ciclo visou abranger a clientela que tivesse freqüentado oAmbulatório de Quimioterapia, por pelo menos seis meses, antes de responder ao questionário.

Os dados foram coletados no período de julho a outubro de 2003 e submetidos à análise estatística descritiva. A associação entre sexo e grupo etário dos entrevistados e as respostas dadas às questões da pesquisa foram avaliadas pelo teste de Qui-quadrado.

\section{RESULTADOS}

Entre os 105 pacientes da amostra estudada, 65\% eram mulheres, 52\% estavam na faixa etária dos 41 a 60 anos; $49 \%$ eram casados e $42 \%$ tinham o ensino fundamental incompleto. A renda familiar declarada por $90 \%$ dos entrevistados variou de um a cinco salários mínimos e $51 \%$ deles participavam com mais de $50 \%$ dos seus rendimentos na composição da renda familiar. Todos os pacientes que participaram deste estudo eram atendidos pelo Sistema Único de Saúde (SUS).

No referente à satisfação com o acesso organizacional, observou-se que 47 pacientes $(44,8 \%)$, esperaram de três a sete dias para iniciar o tratamento, e dentre os motivos apontados por $55,2 \%$ dos usuários, que tiveram um tempo maior de espera, a maioria $(72,4 \%$ ) relatou que a demora decorreu da falta de drogas antineoplásicas e de vagas no Ambulatório.

Em relação às acomodações disponíveis nesse serviço, a maioria dos pacientes referiu que se sentiu confortável nas dependências da sala de espera, do consultório de enfermagem e na sala de administração de quimioterápicos (78\%, 86\% e $94 \%$ respectivamente).

Para $54 \%$ dos pacientes foi possivel manter a continuidade do tratamento, mas, para $46 \%$ foi necessário o adiamento ou interrupção devido à falta de fármacos e/ou vaga no Ambulatório (60,4\%), seguido pela suspensão temporária das aplicações para o manejo dos efeitos colaterais da droga $(20,8 \%)$.

Quanto à satisfação dos pacientes com o atendimento recebido na recepção, pôde ser observado que a grande maioria (95\%) afirmou que sempre era atendida com cordialidade e rapidez (82\%). A maioria deles $(77 \%)$ relatou receber informações das funcionárias sobre as rotinas do setore, $91 \%$ referiram não ter tido dúvidas sobre as informações prestadas. Os pacientes que apontaram dúvidas (9\%) afirmaram ter solicitado esclarecimento adicional que foi satisfatório.

No que diz respeito à consulta de enfermagem, a quase totalidade dos pacientes da amostra (98\%), informou que o tempo utilizado foi suficiente e que as orientações prestadas foram de grande utilidade. O tempo despendido na primeira consulta variou entre 31 a 60 minutos, enquanto que nas de 


\begin{tabular}{|c|c|c|c|c|c|c|c|c|c|c|}
\hline \multirow{2}{*}{$\begin{array}{l}\text { CRITÉRIOS DE AVALIAÇÃO } \\
\text { Comportamento da Enfermeira }\end{array}$} & \multicolumn{2}{|c|}{ Sempre } & \multicolumn{2}{|c|}{ Quase sempre } & \multicolumn{2}{|c|}{ Raramente } & \multicolumn{2}{|c|}{ Nunca } & \multicolumn{2}{|c|}{ Total } \\
\hline & $\mathrm{n}$ & $\%$ & $\mathrm{n}$ & $\%$ & $\mathrm{n}$ & $\%$ & $\mathrm{n}$ & $\%$ & $\mathrm{n}$ & $\%$ \\
\hline Ela o trata pelo nome & 99 & 94,0 & 5 & 5,0 & 1 & 0,9 & 0 & 0 & 105 & 100,0 \\
\hline Mostra-se interessada em ajudá-lo a resolver seus problemas & 99 & 90,0 & 11 & 10,0 & 0 & 0 & 0 & 0 & 105 & 100,0 \\
\hline Deixa-o a vontade para fazer perguntas & 102 & 97,0 & 3 & 2,8 & 0 & 0 & 0 & 0 & 105 & 100,0 \\
\hline Mostra-se apressada em terminar a consulta e fazer anotações & 9 & 8,5 & 2 & 1,9 & 6 & 5,7 & 88 & 83,0 & 105 & 100,0 \\
\hline Mostra-se indiferente em relação aos problemas do paciente & 6 & 5,7 & 0 & 0 & 2 & 1,9 & 97 & 92,0 & 105 & 100,0 \\
\hline Desempenho dos Técnicos e Auxiliares de Enfermagem & $\mathrm{n}$ & $\%$ & $\mathrm{n}$ & $\%$ & $\mathrm{n}$ & $\%$ & $\mathrm{n}$ & $\%$ & n & $\%$ \\
\hline Possuem habilidade para puncionar veias ou cateteres & 90 & 86,0 & 15 & 14,0 & 0 & 0 & 0 & 0 & 105 & 100,0 \\
\hline Observam freqüentemente a administração & 103 & 98,0 & 2 & 1,9 & 0 & 0 & 0 & 0 & 105 & 100,0 \\
\hline São cuidadosos para retirar a agulha no final da administração & 103 & 98,0 & 2 & 1,9 & 0 & 0 & 0 & 0 & 105 & 100,0 \\
\hline Transmitem segurança durante a administração & 95 & 92,0 & 8 & 7,6 & 2 & 1,9 & 0 & 0 & 105 & 100,0 \\
\hline Atendem com rapidez às solicitações dos pacientes & 97 & 92,0 & 8 & 7,6 & 0 & 0 & 0 & 0 & 105 & 100,0 \\
\hline
\end{tabular}

Quadro 1. Opinião dos pacientes sobre o comportamento da enfermeira durante a consulta e desempenho dos técnicos e auxiliares de enfermagem durante a administração dos quimioterápicos. São Paulo, 2004.

seguimento, a duração foi entre 15 a 30 minutos. Estes intervalos de tempo justificam-se pelo fato de que, na primeira consulta, a enfermeira colhe os dados, realiza o exame físico e orienta os pacientes e familiares sobre 0 tratamento e seus efeitos colaterais. Nas de seguimento, o enfoque éa avaliação da situação do paciente, principalmente quanto aos efeitos colaterais apresentados após a quimioterapia e à efetividade das medidas utilizadas para manejá-los, assim como o reforço das mesmas, quando necessário.

Os pacientes referiram ainda, que na grande maioria das consultas realizadas, a enfermeira foi cordial e demonstrou interesse em ajudá-los, promovendo, na opinião deles, um bom relacionamento interpessoal. Reconheceram ainda, que os técnicos/auxiliares de enfermagem realizaram adequadamente os procedimentos para administrar os quimioterápicos, o que contribuiu para que sentissem segurança, atribuindo qualidade ao serviço, conforme pode ser observado no Quadro 1.

Os dados sobre a satisfação dos pacientes e os resultados do atendimento mostraram que $90,3 \%$ deles referiram que as orientações prestadas pela enfermeira sempre os auxiliaram a elucidar situações referentes ao processo de doença/tratamento $(88,3 \%)$ e a controlar os efeitos adversos da quimioterapia, possibilitando controlá-los e manter o bem-estar (82,5\%) sem a necessidade de internações decorrentes dos efeitos colaterais do tratamento.. Somente $2,9 \%$ dos pacientes necessitaram de internação no decorrer do tratamento.

Os dois pacientes, que avaliaram como desnecessária a consulta de enfermagem, relataram o fato de não gostarem de falar sobre seus problemas e que as orientações dadas não os ajudaram a evitar o desconforto gerado pela quimioterapia.

As Figuras 1 e 2 demonstram, que os pacientes avaliaram como muito bom 0 atendimento de enfermagem prestado no Ambulatório e referiram estar muito satisfeitos com o atendimento global recebido no serviço (54\% e $50 \%$ respectivamente). Ressalta-se que entre as sugestões dadas pelos pacientes, cerca de $80 \%$ delas foram referentes a componentes da estrutura, tais como: necessidade de ampliação da área física e aumento do número de profissionais e de recursos materiais. Outros comentários: aproximadamente $20 \%$, foram relacionados ao processo assistencial no sentido de manter os procedimentos realizados e a forma de atendimento que vem sendo prestada.

No que diz respeito ao componente de resultados, foi evidenciado que os pacientes não os relacionaram com o controle dos efeitos adversos, ou mesmo com os benefícios que referiram ter obtido com as informações e cuidados recebidos.

As associações estatisticamente significantes quanto às variáveis sexo e faixa etária não mostraram fatos que pudessem ser considerados de importância para a avaliação da satisfação dos pacientes.

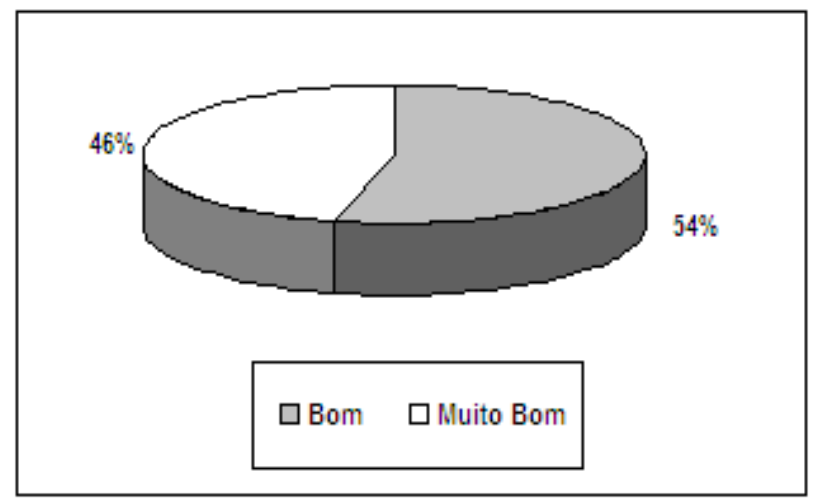

Figura 1. Avaliação atribuída pelos pacientes ao atendimento de enfermagem prestado na consulta e administração de medicamentos. São Paulo, 2004.

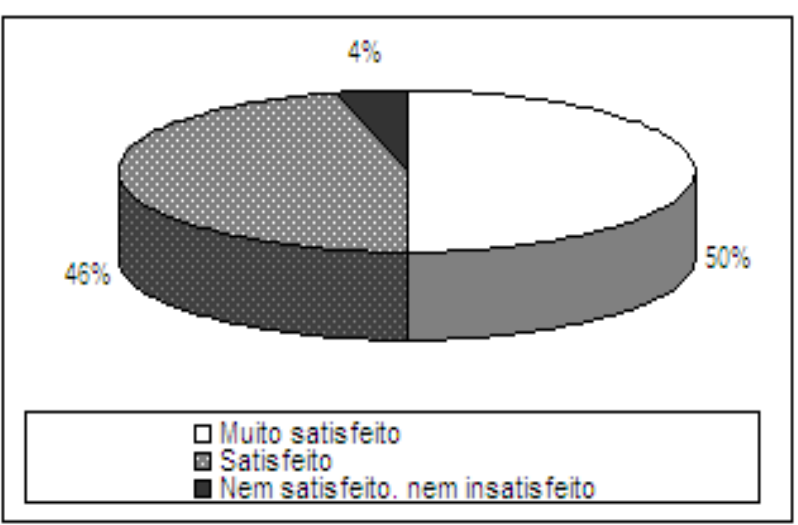

Figura 2. Satisfação do cliente em relação ao atendimento recebido. São Paulo, 2004.

\section{DISCUSSÃO}

A análise de componentes da estrutura mostrou que 0 acesso organizacional para o início do tratamento quimioterápico ocorreu entre 3 a 7 dias para 47 pacientes $(44,8 \%)$ da amostra estudada, tempo necessário para que ocorra o fluxo destinado à liberação das Autorizações de Procedimento de Alto Custo pela Secretaria de Estado da Saúde de São 
Paulo. Contudo, dentre os 58 clientes $(55,2 \%)$ que aguardaram de 8 a mais de 30 dias, 46 pacientes referiram que a demora foi por falta de fármacos $(24)$, de vagas na Instituição (10), ou por estes dois motivos (12), indicando que esse acesso foi dificultado por problemas de estrutura do sistema de saúde.

Refletindo o panorama nacional, no ano de 2005, o câncer foi o diagnóstico médico encontrado em $30 \%$ dos pacientes internados e a segunda causa de morte por doença no HSP, predominando ainda, as ações curativas devido, entre outros fatores, ao fato de o doente ser atendido quando a doença já atingiu estágios mais avançados.

Em decorrência da demanda abranger também pacientes oncológicos procedentes de outros municípios desse Estado e de outras unidades da Federação, o número de clientes encaminhados ao Ambulatório de Quimioterapia tem suplantado a capacidade de atendimento deste serviço, gerando um período maior de espera para início do tratamento quimioterápico. Esta situação confirma a necessidade de melhorar a qualidade da cobertura da assistência oncológica para aumentar o número de pacientes atendidos na sua área regional ${ }^{(9)}$.

Por sua vez, a falta de quimioterápicos até por 15 dias, interrompeu ou adiou a aplicação de protocolos de tratamento no Ambulatório, comprometendo o atendimento prestado, já que estes fármacos são essenciais para a assistência oncológica e melhoria da qualidade de vida dos pacientes atendidos $^{(10,11)}$.

O principal problema evidenciado no sistema de saúde do Município de São Paulo é o acesso ao serviço e, a solução apontada consiste na reestruturação do sistema hospitalar onde os quatro grandes hospitais da cidade seriam responsáveis pelo atendimento dos moradores da região a que pertencem e, para tanto, o Município e o Estado deveriam atuar de forma integrada na política de atendimento do SUS ${ }^{(12)}$.

Entre os aspectos que foram relacionados como geradores de insatisfação destacam-se os referentes à estrutura que, para serem solucionados, dependem tanto de modificações no próprio sistema de saúde, como de decisões dos gestores da Instituição, realizando modificações que diminuam as limitações encontradas no Ambulatório.

No que se refere à avaliação positiva das acomodações dos diversos ambientes físicos do Ambulatório, por parte dos usuários, destaca-se que, as instalações deste serviço, reformadas em 1996, atenderam as normas referentes à estrutura e funcionamento das centrais de quimioterapia. Devido às novas normas da ANVISA para serviços de quimioterapia, a área física do referido Ambulatório necessita ser ampliada, principalmente, nos setores de preparo e de administração de quimioterápicos, para estar em conformidade com a nova legislação $0^{(13)}$.

Em relação ao processo assistencial, a interação profissional/cliente indicou dois elementos que influenciaram a avaliação positiva da qualidade do serviço: a capacidade técnica do profissional, que depende do seu conhecimento e julgamento utilizados nas decisões estratégicas apropriadas para prestar os cuidados ao paciente e da sua competência para a implementação desse processo; e, o adequado relacionamento interpessoal, que é vital, por englobar a afabilidade e interesse dos prestadores da assistência para com os pacientes e o incentivo para que estes decidam participar ativamente no tratamento e cuidados propostos.

$O$ adequado relacionamento interpessoal entre enfermeira e paciente permite, não só propiciar a identificação das necessidades de cuidados, mas também o esclarecimento dos possíveis efeitos do tratamento e a maneira de administrá-los, contribuindo para diminuir a ansiedade e aumentar a adesão ao tratamento. Ressalta-se que, para tal, a enfermeira precisa desenvolver sua habilidade em comunicação e lembrar que a tecnologia se faz importante quando não se esquece 0 aspecto humano e que o bom relacionamento entre cliente e prestador de serviço é um diferencial na qualidade da assistência(14-16).

Um importante indicador da qualidade do cuidado de enfermagem se expressa pela manutenção das ações da enfermeira centradas na orientação dos pacientes, que intensificam a comunicação dela com o cliente e seus familiares $^{(14-17) \text {. }}$

Estas intervenções educativas, no serviço avaliado, são desenvolvidas preponderantemente durante a consulta com a enfermeira, o que leva a reafirmar a importância da manutenção da qualidade dessa atividade, que propicia também, um espaço que garante a privacidade e a oportunidade para que o paciente expresse suas necessidades, preocupações e dúvidas.

Considera-se, portanto, que a informação oportuna e confiável é a matéria-prima no relacionamento da enfermeira com os pacientes e familiares, que precisam da informação para se sentir seguros e protegidos.

No que se refere à satisfação com os aspectos técnicos do cuidado, infere-se que é resultante do investimento das enfermeiras na capacitação contínua dos profissionais de nível técnico desse serviço para o desempenho qualificado das atividades envolvidas na administração dos quimioterápicos antineoplásicos. Os resultados deste desempenho podem ser evidenciados pelas baixas taxas de incidência de extravasamentos de drogas citostáticas nesse Ambulatório(18).

A análise do componente de resultados, expresso neste estudo pela adesão ao tratamento, número de hospitalizações ocorridas por complicações relacionadas aos efeitos adversos da quimioterapia, e a verbalização sobre os benefícios auferidos com as informações e cuidados recebidos, evidencia que a assistência prestada é de qualidade, uma vez que mais da metade dos pacientes (54\%) não interrompeu o tratamento e somente três pacientes $(2,9 \%)$ necessitaram de internação no decorrer do mesmo.

É importante destacar que a interrupção do tratamento, ocorrida em $46 \%$ da amostra, foi motivada por fatores estruturais, independentes, portanto, da esfera de decisão dos pacientes.

Ressalta-se ainda, que os atributos que prevaleceram na avaliação positiva dos usuários do serviço com 0 atendimento recebido foram 0 ambiente acolhedor e o bom relacionamento entre profissionais e pacientes.

É reconhecido que nem sempre a satisfação do usuário guarda uma relação com a boa ou má qualidade do atendimento, enfocando-se para esse julgamento os critérios técnicos. Os leigos fazem seus julgamentos a partir das relações com o trato humanístico que recebem, tais como: respeito, comunicação clara, possibilidade de decidir frente às ações de tratamento, tolerância e compreensão que são demonstradas pela equipe que os assiste. Assim, as pessoas que pertencem a grupos de menor condição sócioeconômica, como os usuários do serviço avaliado, utilizam critérios de natureza emocional, como afabilidade, para estabelecer a sua avaliação acerca de um determinado serviço. Já as classes de maior condição sócioeconômica avaliam os serviços prestados considerando, também, os aspectos técnicos envolvidos no cuidado(19).

\section{CONCLUSÃO}

Em que pese o fato do acesso organizacional ter ocorrido em período maior do que sete dias para $58 \%$ dos 105 pacientes da amostra estudada e que para 33 usuários, o tratamento foi interrompido por fatores estruturais, a grande maioria (96\%) demonstrou satisfação com o atendimento recebido no Ambulatório avaliado. Os atributos valorizados foram os relacionados ao acolhimento com que os pacientes foram recebidos neste serviço.

Acredita-se que os resultados desta pesquisa, se aplicados na prática, poderão contribuir para melhoria da qualidade da assistência prestada no Ambulatório estudado, dentro de uma concepção de transformação que parte da consideração da opinião dos usuários do serviço, como propõe o grupo de avaliadores de quarta geração e que reforce o conceito de humanização do SUS, abrangendo as relações entre os profissionais de saúde e a própria instituição, na busca de condições mínimas de trabalho que dêem suporte ao processo de atendimento humanizado. 


\section{REFERÊNCIAS}

1. Diacou R, Silva NT, Maia AC. Qualidade do serviço hospitalar: uma abordagem sistêmica. Cadernos - Centro Universitário São Camilo 2004; 10(3): 88-105.

2. Silva SH. Controle da qualidade assistencial de enfermagem implementação de um modelo (tese). São Paulo (SP): Escola de Enfermagem, USP; 1994.

3. Furtado JP. Um método construtivista para a avaliação em saúde Cien Saúde Col 2001; 6(1): 65-181.

4. Adami NP, Yoshitome AY. Métodos de avaliação de resultados da assistência de enfermagem. Rev Bras Enferm 2003; 56(1): $52-6$.

5. Bittar OJNV. Indicadores de qualidade e quantidade em saúde. Rev Adm Saúde 2001; 3(12): 21-8.

6. Donabedian A. The seven pillars of quality. Arch Pathol Lab Med 1990; 114.

7. Agnol CMD, Trench MH. Grupos focais como estratégia metodológica em pesquisas na enfermagem. Rev Gaucha Enferm 1999; 20(1): 1-158

8. Sitzia J, Wood N. Patients satisfaction with cancer chemotherapy nursing: a review of the literature. Intern J Nursing Studies 1998; 35: 1 -12.

9. Kligerman J. Assistência oncológica e incorporação tecnológica. Rev Bras Cancerol 2001, 47(3): 239-43.

10. Lemme AC, Noronha G, Resende JB. A satisfação do usuário em hospital universitário. Rev Saúde Publ 1991; 25(1): 41-6.

11. Machado dos Santos SC. A política de fármacos eleva a política de saúde. In: Negri B, Viana ALD, organizadoras. O sistema único de saúde em dez anos de desafio. São Paulo (SP): Sobravime/Cealag; 2002. p. 353-87.

12. Carvalho MC. Dez soluções para a cidade: especialistas sugerem enfoques inovadores. Saúde pública - Divisão por regiões racionaliza sistema. Folha de São Paulo 31 out 2004; 9.

13. Brasil. Ministério da Saúde. Anvisa. Regulamento técnico de funcionamento dos serviços de terapia antineoplásica. Resolução RDC $n^{0} 220$ de 21 de setembro de 2004. Brasília (DF): Ministério da Saúde 2004

14. Cadah L. Avaliação da qualidade da assistência de enfermagem sob a ótica da satisfação dos pacientes (dissertação). São Paulo (SP): Escola de Enfermagem, USP; 2000.

15. Ellis C, Evans BD, Mak D, Mitchell P, Melville P, Stone C, et al. Patient assessment of a combined medical and nursing preparation to cytotoxic chemotherapy. Sup Care Cancer 1993; 1: 209-13.

16. Oermann M H, Templim T. important attributes of quality health care: consumer perspectives. J Nurs Scholarship 2000; 32(2).

17. Bisagni C, Mauro MYC. Unidade de imagem: a consulta de enfermagem na qualificação do processo de trabalho. Rev Enf Esc Anna Nery 2001; 5(3): 365-75.

18. Adami NP, Gutiérrez MGR, Fonseca SM, Almeida EPM. Riks management of extravasations of citostatic drugs at the adult chemotherapy outpatient clinic of a University Hospital. J Clin Nurs. No prelo 2005.

19. Adami NP. Acesso, utilização e aceitação dos serviços de dermatologia de um centro de saúde escola sob o modo de ver dos hansenianos. Rev Latino-am Enfermagem 1993; 2(1): 53-67. 